\title{
Obstructions to Pin Structures on Kleinian Manifolds
}

\author{
L. J. Alty and A. Chamblin \\ Department of Applied Mathematics and Theoretical Physics, \\ Silver Street, Cambridge CB3 9EW, England
}

\begin{abstract}
We develop various topological notions on four-manifolds of Kleinian signature $(--++)$. In particular, we extend the concept of 'Kleinian metric homotopy' [1] to non-orientable manifolds. We then derive the topological obstructions to pinKlein cobordism, for all of the pin groups. Finally, we discuss various examples and applications which arise from this work.
\end{abstract}

\section{Introduction}

Let $M$ be any smooth four-manifold,then we say that a metric $g$ on $M$ is of Kleinian signature if it has signature $(--++)$. In recent work [1], we derived the topological obstruction to spin-Klein cobordism, and in this paper, we treat the interesting and non-trivial problem of extending this work to non-orientable manifolds.

An orientable Kleinian manifold $(M, g)$ has orthonormal frame bundle $\tau(M)$ with structure group $S O(2,2)$. We say that $M$ admits a spin structure if and only if there exists a $2-1$ covering, $\bar{\tau}(M) \longrightarrow \tau(M)$, such that the following diagram commutes:

$$
\begin{aligned}
& \operatorname{Spin}(2,2) \longrightarrow \bar{\tau}(M) \longrightarrow M \\
& \downarrow^{2-1} \quad \downarrow^{2-1} \quad \downarrow \quad \text { identity } \\
& S O(2,2) \quad \longrightarrow \quad \tau(M) \longrightarrow M
\end{aligned}
$$


where $\operatorname{Spin}(2,2)$ is the double cover of $S O(2,2)$.

When $M$ is non-orientable, one cannot reduce the tangent bundle $\tau(M)$ to a bundle with structure group $S O(2,2)$; indeed, $\tau(M)$ can only be reduced to a bundle with structure group $O(2,2)$. In analogy with the above construction, we then seek all groups $\bar{O}(2,2)$ which are double covers of $O(2,2)$; that is, we seek all groups $\bar{O}(2,2)$ so that the following sequence is exact:

$$
1 \longrightarrow \mathbb{Z}_{2} \longrightarrow \bar{O}(2,2) \longrightarrow O(2,2) \longrightarrow 1 \text {. }
$$

In fact (see eg. [2,3]), there are eight distinct such groups which are double covers of $O(2,2)$. Following Dabrowski [2], we will call these covers the pin groups for Kleinian signature and write them as

$$
h^{a, b, c}: \operatorname{Pin}^{a, b, c}(2,2) \longrightarrow O(2,2)
$$

with $a, b, c \in\{+,-\}$.

In order to interpret the signs $a, b$ and $c$, it is convenient to keep some of the terminology from Lorentzian geometry. Thus we will say that a vector $v \in T_{p}(M)$ is spacelike if $g(v, v)>0$, timelike if $g(v, v)<0$, and null if $g(v, v)=0$.

Now we recall that $O(2,2)$ is not path connected; there are four components, given by the identity connected component, $O_{0}(2,2)$, and the three components corresponding to 'space' inversion $S$, 'time' inversion $T$, and the combination of these two, $S T$ (i.e., $O(2,2)$ decomposes into a semi-direct product 円, $\left.O(2,2) \simeq O_{0}(2,2) \odot\left(\mathbb{Z}_{2} \times \mathbb{Z}_{2}\right)\right)$. By 'space' inversion, we mean reflection about a plane, $v^{\perp}$, perpendicular to some spacelike vector $v$; likewise, 'time' inversion is reflection about a plane perpendicular to a timelike vector. The signs of $a, b$, and $c$ then correspond to the signs of the squares of the elements in $\operatorname{Pin}^{a, b, c}(2,2)$ which cover space inversion, time inversion, and a combination of the two respectively.

Indeed, with these conventions we can write out the explicit form of the groups $\operatorname{Pin}^{a, b, c}(2,2)$; they are given by the semi-direct product [2]

$$
\operatorname{Pin}^{a, b, c}(2,2) \simeq \frac{\left(\operatorname{Spin}_{0}(2,2) \odot C^{a, b, c}\right)}{\mathbb{Z}_{2}}
$$

\footnotetext{
${ }^{1}$ ie. $O(2,2)$ is the disjoint union

$O(2,2) \simeq O_{0}(2,2) \cup S\left(O_{0}(2,2)\right) \cup T\left(O_{0}(2,2)\right) \cup S T\left(O_{0}(2,2)\right)$.
} 
where the $C^{a, b, c}$ are the four double coverings of $\mathbb{Z}_{2} \times \mathbb{Z}_{2}$, as outlined in ([2], [3]).

On surveying the above constructions, one might wonder why we are concerned with developing the obstruction theory for $\operatorname{Pin}^{a, b, c}(2,2)$ fibre bundles, since with $\pi_{1}(O(2,2)) \simeq \mathbb{Z} \times \mathbb{Z}$, and so there is no way that the pin bundles will allow us to represent all of the information contained in the tangent bundle in a simply-connected manner [3]. Indeed, if we wished to represent the information in $\tau(M)$ in a simply-connected manner, we would seek a bundle $\xi(M)$, with structure group $\hat{O}$ given by the exact sequence

$$
1 \longrightarrow \pi_{1}(O(2,2)) \simeq \mathbb{Z} \times \mathbb{Z} \longrightarrow \hat{O} \longrightarrow O(2,2) \longrightarrow 1
$$

wheras the pin groups are given by the short exact sequence

$$
1 \longrightarrow \mathbb{Z}_{2} \longrightarrow \operatorname{Pin}^{a, b, c}(2,2) \longrightarrow O(2,2) \longrightarrow 1 \text {. }
$$

It follows that any pin bundle $P(M)$ (ie. any bundle with fibre $\operatorname{Pin}^{a, b, c}(2,2)$ ) will not represent information in a simply-connected way. This means that at a point $p \in M$ there exist paths $\rho_{1}, \rho_{2} \in \operatorname{Pin}^{a, b, c}(2,2)$ which might act on the fibre $\left.P(M)\right|_{p}$ equivalently (in the sense that, for $\left.x \in P(M)\right|_{p}, \rho_{1}(x)=\rho_{2}(x)$ ), but with the property that $\rho_{1}$ and $\rho_{2}$ (viewed as curves in $\operatorname{Pin}^{a, b, c}(2,2)$ ) are not homotopic. Indeed, one sees that the 'particles' corresponding to such a simply connected representation could have aribitrary fractional statistics and would be 'anyons' [4]. The point is that for both Riemannian and Lorentzian signature (in four dimensions) one obtains a simply connected representation of tangent bundle information by passing to a fermionic (or pin) bundle; it is only for Kleinian signature that this does not work and one needs to introduce some anyonic structure.

At any rate, these mathematical considerations aside, the primary reason why we wish to understand the obstructions to pin bundles comes from physics. In particular, recent work on signature change (see eg. [5-8]) has suggested that we should allow for regions of non-Lorentzian signature in our description of nature. The idea is that we should consider manifolds of the form $M \cong M_{L} \cup M_{R}$ and $M^{\prime} \cong M_{L}^{\prime} \cup M_{K}^{\prime}$, where (for example) $M_{R}$ is some Riemannian manifold, $M_{L}$ and $M_{L}^{\prime}$ are some Lorentzian manifolds, and $M_{K}^{\prime}$ is some Kleinian manifold (where $\emptyset \neq \partial M_{L}=\Sigma=\partial M_{R}$ and $\partial M_{L}^{\prime}=\Sigma^{\prime}=\partial M_{K}^{\prime} \neq \emptyset$, so that the signature is said to 'change' across the three-surfaces $\Sigma$ and $\Sigma^{\prime}$, which are generically taken to be stationary with 
respect to the ambient four-metrics). If one is going to assert that there are 'regions' of Kleinian signature, then one should try to make sense of field theory [8] in signature $(--++)$. In particular, one must make sense of the Dirac equation:

$$
i \gamma^{a} \partial_{a} \psi=0
$$

where $\left\{\gamma^{a}, \gamma^{b}\right\}=2 g^{a b}$. Solutions of (1) will generically take values in some pin bundle, and so this is one reason the cobordism problem is so interesting. There are other physical applications for Kleinian signature manifolds, including the $N=2$ superstring theory [9]. In this theory, the Weyl anomaly cancels provided the string propagates in a four-dimensional target space, and if the worldsheet has Lorentzian signature then the target space must have Kleinian signature.

\section{Klein metric homotopy}

We wish to understand the topology of Klein metrics on four-manifolds which are not necessarily orientable. The fundamental result we begin with is the following lemma of Steenrod [10]

Lemma 1. Let $M$ be a smooth four-manifold without boundary. Then $M$ admits a globally defined (non-singular) Klein metric if and only if there exists a globally defined (non-singular) field of 2-planes on $M$.

In [1] we restricted our consideration to fields of oriented 2-planes; that is, since we were only considering orientable four-manifolds $M$, we assumed that there were no closed loops, $\gamma$, in $M$ around which we could propagate a 2-plane, $P$, and end up with the opposite orientation (of the plane $P$ ). Technically, this meant that we assumed our plane fields to be sections of the fibre bundle over $M$ with fibre $G_{2,4} \cong S^{2} \times S^{2}$, where $G_{2,4}$ is (by definition) the set of oriented 2-planes in $\mathbb{R}^{4}$.

If, however $M$ is non-orientable then there will exist loops in $M$ such that, when we propagate plane fields around them, the orientation of the planes will be reversed as shown in Fig. 1). In this case, we must now define a plane field to be a section of the bundle of unoriented planes. That is, let

$\tilde{G}_{2,4}$ denote the set of unoriented plane fields in $\mathbb{R}^{4}$. Then a field of 2-planes is a section of the fibre bundle with fibre $\tilde{G}_{2,4}$. 
This situation is reminiscent of what happens in Lorentzian geometry when one passes from the study of time-orientable geometries to non-timeorientable geometries $([3,11])$; there, one passes from a vector field to a 'line' field (i.e., an undirected or unoriented vector field). However, the analogy should not be taken too far. In Lorentzian geometry, non-time-orientability is a serious matter since it implies that we have no local notion of an 'arrow of time', and thus many of our thermodynamical notions become tenuous.

For Kleinian geometry, there is no 'arrow of time', since at a point there is a '2-plane's' worth of timelike directions. In other words, when we identify a Kleinian metric $g$ with a 2-plane field $P$, we can essentially take $P$ to be the plane spanned by the set of timelike directions (at each point). Indeed, in this paper we will always take $P$ to be a 'timelike' plane field. It follows that there is no sensible notion of causal structure, or of causality, in a Kleinian manifold. In fact, there are 'closed timelike curves' through every point, as shown in Fig. 2. One can always just 'rotate' into one's own past. Indeed, 'time' itself has a chirality (i.e., the orientation of the plane field $P$ ). These considerations, if anything, make it clear that orientability is less relevant in Kleinian geometry than it is in Lorentzian geometry.

Now that we have made sense of what we mean by a 'field of unoriented 2-planes', we need to consider the obstruction to constructing such a 2-plane field which is globally non-singular. To do this, we first examine the details of Hirzebruch and Hopf's [12] original treatment of the subject.

Let $M$ be an oriented smooth manifold, and $P$ some field of oriented 2-planes on $M$. Generically, $P$ will be singular on a finite set of points, $\left\{p_{1}, p_{2}, \ldots, p_{n}\right\}$ in $M$. Each singularity $p_{i}$ of $P$ will have associated to it an index. The index of the singularity $p_{i}$ is the homotopy type of the map (defined by the plane field $P$ ) from a little three-sphere, $S^{3}\left(p_{i}\right)$, surrounding $p_{i}$ to $G_{2,4}$. Such homotopy classes are in one-to-one correspondence with elements of $\pi_{3}\left(G_{2,4}\right) \simeq \mathbb{Z} \oplus \mathbb{Z}$. Thus, the index of $P$ at $p_{i}$ is classified by a pair of integers (intuitively, this index measures the 'winding' of $P$ as one moves around $S^{3}\left(p_{i}\right)$ ). We denote the index of $P$ at $p_{i}$ by the symbol $\operatorname{ind}\left(P, p_{i}\right)$. Since there are generically finitely many singular points $p_{i}$, one can form the index of $P$ on $M$ :

$$
\text { index of } P \text { on } M=\sum_{i=1}^{n} \operatorname{ind}\left(P, p_{i}\right) \text {. }
$$

In [12] Hirzebruch and Hopf developed a result which gives the exact form 
of the index for orientable manifolds without boundary. The statement of their result is as follows:

Let $M$ be an oriented compact four-manifold without boundary. Let $H$ denote the free abelian group $H^{2}(M, \mathbb{Z})$ /torsion subgroup, and let $S$ denote the intersection pairing on $H$ defined by the cup-product (ie. $S$ defines a map from $H \otimes H$ to $\mathbb{Z}$ by taking the cup-product of elements in $H$ and evaluating them on the fundamental orientation class of $M)$. Define the coset $W \subseteq H / 2 H$ by $w \in W$ if $S(w, x)=S(x, x) \bmod 2$ for all $x \in H$. Finally, let $\Omega$ denote the set of integers $\{S(w, w) \mid w \in W\}$, then we have $[12,13]$

Theorem 1. Let $M$ be an oriented compact four-manifold without boundary. Then $M$ has a field of 2-planes with finite singularities. The total index of such a field is given by a pair of integers $(a, b)$. The following integers and only these, occur as the index for some plane field on $M$ :

$$
a=\frac{1}{4}(\alpha-3 \sigma-2 \chi), b=\frac{1}{4}(\beta-3 \sigma+2 \chi)
$$

where $\alpha, \beta \in \Omega, \chi=\chi(M)$ denotes the Euler number of $M$, and $\sigma=\sigma(M)$ denotes the Hirzebruch signature of $M$.

We wish to be able to calculate the index of a plane field on a nonorientable manifold, and thus it is instructive to examine the proof of this theorem to see exactly which steps are invalid when one passes to the nonorientable case.

To begin with, recall that the Stiefel manifold, $V_{2,4}$, is defined to be the set of oriented dyads $\left\{v_{1}, v_{2}\right\}$ in $\mathbb{R}^{4}$. It is clear that any dyad $\left\{v_{1}, v_{2}\right\}$ induces a plane $P$ (ie. $P$ is spanned by $v_{1}$ and $v_{2}$ ) and so we have the inclusion

$$
\varphi: V_{2,4} \longrightarrow G_{2,4} \text {. }
$$

Likewise, we can consider the Stiefel manifold $\tilde{V}_{2,4}$ of unoriented dyads in $\mathbb{R}^{4}$; then we have the inclusion

$$
\tilde{\varphi}: \tilde{V}_{2,4} \longrightarrow \tilde{G}_{2,4}
$$

Now, in [12] the construction begins by considering the skeleton [14] of $M$. Let $M^{1}$ denote the 1 -skeleton, $M^{2}$ the 2 -skeleton, etc., then we always

\footnotetext{
${ }^{2}$ ie. a dyad is a pair of vectors.
} 
can put a dyad field $\left\{v_{1}, v_{2}\right\}$ (a section of the fibre bundle with fibre $V_{2,4}$ or $\tilde{V}_{2,4}$ ) on the 2 -skeleton. If $M$ is oriented, we can take this dyad field to be oriented; if $M$ is not oriented, we will generically have to take the dyad field to be unoriented. We then want to extend the dyad field on $M^{2}$ to a dyad field on $M^{3}$. In the oriented case, the obstruction to doing this is $w_{3}(M)$ [14], the third Stiefel-Whitney class of $M$. Of course, for a compact oriented $M, w_{3}(M)=0$, and so one is able to conclude [12] that the obstruction to extending a plane field to all of $M$ must be an element of $H^{4}\left(M ; \pi_{3}\left(G_{2,4}\right)\right) \simeq H^{4}(M ; \mathbb{Z} \oplus \mathbb{Z}) \simeq H^{4}(M ; \mathbb{Z}) \oplus H^{4}(M ; \mathbb{Z}) \simeq \mathbb{Z} \oplus \mathbb{Z}$.

When $M$ is not oriented, it might be thought that there is some other obstruction to extending a dyad field (and thus a plane field) from $M^{2}$ to $M^{3}$. However, this is not the case. Although Wu's formula [14] shows that $w_{3}(M)$ can be non-vanishing for $M$ non-oriented, $w_{3}(M)$ will no longer be the obstruction to extending the field $P$ to $M^{3}$ since we are now allowing the plane field to be unoriented. Indeed, the obstruction to this extension vanishes as long as $P$ is a section of a $\tilde{G}_{2,4}$ bundle. It follows that the obstruction to extending an unoriented plane field $P$ over an unoriented four-manifold $M$ is an element of $H^{4}\left(M ; \pi_{3}\left(\tilde{G}_{2,4}\right)\right)$. But $G_{2,4}$ is the $2-1$ cover of $\tilde{G}_{2,4}$ and so

$$
\pi_{3}\left(G_{2,4}\right) \simeq \pi_{3}\left(\tilde{G}_{2,4}\right) \simeq \mathbb{Z} \oplus \mathbb{Z}
$$

Thus, in both the oriented and non-oriented cases, the obstruction to extending $P$ over $M$ is an element of $H^{4}(M ; \mathbb{Z} \oplus \mathbb{Z})$.

At first, this may seem strange, since for a non-orientable manifold $M$, $H^{4}(M ; \mathbb{Z}) \simeq \mathbb{Z}_{2}$, and so the total index would seem to be an element of

$$
H^{4}(M ; \mathbb{Z} \oplus \mathbb{Z}) \simeq H^{4}(M ; \mathbb{Z}) \oplus H^{4}(M ; \mathbb{Z}) \simeq \mathbb{Z}_{2} \oplus \mathbb{Z}_{2},
$$

and so the index is only defined 'up to parity'. However, as we shall see, the parity of the index is the only thing relevant in the construction of our obstructions.

Before continuing with the derivation of the form of the index, it is necessary to recall some elementary topological objects which we will make use of later. To begin with, we have that a manifold $M$ admits a globally defined metric of Kleinian signature if and only if the tangent bundle of $M(\tau(M))$ can be globally decomposed into the Whitney sum

$$
\tau(M) \simeq \tau^{+} \oplus \tau^{-}
$$


where $\tau^{+}$is the subbundle of $\tau(M)$ generated by 'spacelike' vectors and $\tau^{-}$is the subbundle generated by 'timelike' vectors. Let $w_{1}(M)=w_{1}(\tau(M))$ denote the first Stiefel-Whitney class of $M$. As is well known [14], $w_{1}(M)=0$ if and only if $M$ is orientable. Since $w_{1}$ is a 1-cochain, this means that $M$ is orientable if and only if there are no closed loops, $\gamma \in M$, such that $w_{1}[\gamma] \neq 0$. Under the Whitney sum, $w_{1}(\tau(M))$ can be decomposed as

$$
w_{1}(\tau(M))=w_{1}\left(\tau^{+}\right)+w_{1}\left(\tau^{-}\right) .
$$

We shall adopt the notation $w_{1}^{+}=w_{1}\left(\tau^{+}\right)$and $w_{1}\left(\tau^{-}\right)=w_{1}^{-}$. Thus, $M$ is space-orientable if and only if $w_{1}^{+}=0$, and time-orientable if and only if $w_{1}^{-}=0$. Note that if there exists some loop, $\gamma \in M$, such that $M$ is neither space nor time-orientable, then $M$ is orientable since $w_{1}(\tau(M))=$ $w_{1}^{+}+w_{1}^{-}=1+1=0 \bmod 2$. (We always count mod 2 since these cochains always take values in $\left.\mathbb{Z}_{2}[3]\right)$. As we shall see, $w_{1}^{+}$and $w_{1}^{-}$are critical components of the obstructions to all of the pin structures.

Let us denote the second Stiefel-Whitney class, $w_{2}(M)=w_{2}(\tau(M))$. Recall that this class vanishes if and only if $M$ admits a spin structure.

Now as in [3], we can apply Wu's formula and obtain the identity:

$$
\left(w_{2}(M)+w_{1}(M) \smile w_{1}(M)\right) \smile x_{2}=x_{2} \smile x_{2} \quad \text { for any } x_{2} \in H^{2}\left(M ; \mathbb{Z}_{2}\right),
$$

where ' $\smile$ ' is the cup product [14]. Since we are allowing $M$ to be nonorientable, we work in $\mathbb{Z}_{2}$-coefficients and write the intersection pairing

$$
h: H_{2}\left(M ; \mathbb{Z}_{2}\right) \times H_{2}\left(M ; \mathbb{Z}_{2}\right) \longrightarrow \mathbb{Z}_{2}
$$

This is defined by $h(x, y)=x \cdot y=\left(x_{2} \smile y_{2}\right) \frown w_{1}$, where $x_{2}, y_{2} \in H^{2}\left(M ; \mathbb{Z}_{2}\right)$ satisfy $x_{2} \frown w=x$ and $y_{2} \frown w=y, w \in H^{4}\left(M ; \mathbb{Z}_{2}\right)$ is the fundamental homology class and ' $\supset$ ' denotes cap product. Taking the dual of equation (4) yields the intersection pairing on $H^{2}\left(M ; \mathbb{Z}_{2}\right)$.

As we saw in [3], the following result of Kervaire and Milnor [15] holds even for non-orientable $M$ :

Lemma 2. Let $M$ be a smooth four-dimensional manifold. Let $u(\partial M)$ (the mod 2 Kervaire semi-characteristic) be given by

$$
u(\partial M)=\operatorname{dim}_{\mathbb{Z}_{2}}\left(H_{0}\left(\partial M ; \mathbb{Z}_{2}\right) \oplus H_{1}\left(\partial M ; \mathbb{Z}_{2}\right)\right) \bmod 2
$$


Then the rank of the intersection pairing, $h$, satisfies

$$
\operatorname{rank}(h)=(u(\partial M)+e(M)) \bmod 2
$$

where $e(M)$ is the Euler number of $M$.

It is now easy to check [3] that $\operatorname{rank}(h)=0$ if and only if

$$
w_{2}+w_{1} \smile w_{1}=0 \bmod 2 .
$$

Combining this observation with Lemma 2 then yields

Lemma 3. Let $M$ be a smooth four-dimensional manifold with tangent bundle $\tau(M)$. Then

$$
\begin{gathered}
w_{2}(\tau(M))+w_{1}(\tau(M)) \smile w_{1}(\tau(M))=0 \\
\Longleftrightarrow \\
(u(\partial M)+e(M)) \bmod 2=0
\end{gathered}
$$

We have now developed enough mathematical machinery to calculate the index of a non-orientable plane field on a general (not necessarily oriented) four-dimensional manifold $M$. Our basic strategy is the following: If $M$ is oriented, we are done (we just apply the Hirzebruch-Hopf result, Theorem $1)$. If $M$ is not orientable, we pass to the oriented double cover, $\tilde{M}$, of $M$ and apply Theorem 1 on $\tilde{M}$. We then 'push down' the plane field $\tilde{P}$ on $\tilde{M}$, under the projection $\pi: \tilde{M} \longrightarrow M$, and deduce the form of the index of $P=\pi^{*}(\tilde{P})$ on $M$. Since any plane field $P$ on $M$ can be so obtained, we thus derive the general form of the index on $M$.

Suppose then we are given some smooth four-dimensional manifold $M$ without boundary, with Klein metric ' $g_{K}$ ' defined on $M$. As we have seen, the metric corresponds to some two-plane field $P$ on $M$. The singularities of the metric $g_{K}$ therefore correspond to the singularities of the plane field $P$. Construct the (oriented) $2-1$ cover over $M$, denoted $\tilde{M}$, with projection $\pi: \tilde{M} \longrightarrow M$. Now lift the plane field $P$ (which will generally be a section of a $\tilde{G}_{2,4}$ bundle, $B$, over $M$ ) to a plane field $\tilde{P}$ over $\tilde{M}$ (where $\tilde{P}$ will now 
be a section of a $G_{2,4}$ bundle, $\tilde{B}$, over $\left.\tilde{M}\right)$. Since $\tilde{M}$ is oriented, we know the form of the total index of $\tilde{P}$ on $\tilde{M}$ is, by Theorem 1 ,

$$
\operatorname{ind}(\tilde{P}, \tilde{M})=\frac{1}{4}(\alpha-3 \sigma-2 \chi, \beta-3 \sigma+2 \chi)
$$

where $\sigma=\sigma(\tilde{M}), \chi=\chi(\tilde{M})$. As in [1], we find that

$$
\begin{aligned}
& \alpha=\sigma(\tilde{M}) \bmod 8, \\
& \beta=\sigma(\tilde{M}) \bmod 8,
\end{aligned}
$$

hence

$$
\begin{aligned}
& \alpha-\sigma=8 n, n \in \mathbb{Z}, \\
& \beta-\sigma=8 m, m \in \mathbb{Z},
\end{aligned}
$$

so the index becomes

$$
\operatorname{ind}(\tilde{P}, \tilde{M})=\frac{1}{4}(8 n-2(\sigma(\tilde{M})+\chi(\tilde{M})), 8 m-2(\sigma(\tilde{M})-\chi(\tilde{M})))
$$

where $m$ and $n$ are some integers. It follows that we must have

$$
\sigma(\tilde{M})=\chi(\tilde{M}) \bmod 2 .
$$

We must now determine how the parity of $\chi(\tilde{M})$ is related to the parity of $\chi(M)$. To do this we introduce a new invariant:

Definition: Let $M$ be a smooth four-dimensional manifold with boundary $\partial M \cong \Sigma_{1} \cup \Sigma_{2} \cup \ldots \cup \Sigma_{n}$ the disjoint union of finitely many (not necessarily orientable) closed three-manifolds. Let $\partial \tilde{M} \cong \tilde{\Sigma}_{1} \cup \tilde{\Sigma}_{2} \cup \ldots \cup \tilde{\Sigma}_{n}$ denote the oriented double cover of $\partial M$. Then we define the element $U(\partial M) \in \mathbb{Z}_{2}$ by the formula

$$
U(\partial M)=(u(\partial M)-u(\partial \tilde{M})) \bmod 2
$$

Thus, $U(\partial M)$ measures (modulo 2) the total number of torsion generators of $H_{1}(\partial M)$ which are 'destroyed' when we pass to the double cover.

\footnotetext{
${ }^{3}$ That is, $\tilde{\Sigma}_{i}$ is the oriented double cover of $\Sigma_{i}$ for each $i$, and $\partial \tilde{M}$ is the disjoint union of the $\tilde{\Sigma}_{i}$.
} 
For example, suppose that $\partial M \cong S^{1} \times \mathbb{R P}^{2}$, then $u(\partial M)=1$ since $\mathbb{R} \mathbb{P}^{2}$ has a torsion generator; and $\partial \tilde{M} \cong S^{1} \times S^{2}$ so $u(\partial \tilde{M})=0$, hence $U(\partial M)=1$. Similarly all the torsion generators are destroyed if we take $\partial M \cong \mathbb{R P}^{3}$.

On the other hand, there are torsion generators which do not completely 'unwrap'. For example, take $\partial M \cong S^{1} \times K$, where $K$ denotes the Klein bottle. We then find that $u(\partial M)=0$, where one of the $H_{1}(\partial M)$ factors is torsion, and that $\partial \tilde{M} \cong S^{1} \times T^{2}$ has $u(\partial \tilde{M})=0$. Hence $U(\partial M)=0$, which makes sense since the torsion generator in $K$ lifts to a non-trivial loop in $T^{2}$.

We must also introduce another new invariant, which we define as follows:

Definition: Let $M$ be a smooth four-manifold, with or without boundary. Then we define the element $\delta(M) \in \mathbb{Z}_{2}$ as follows:

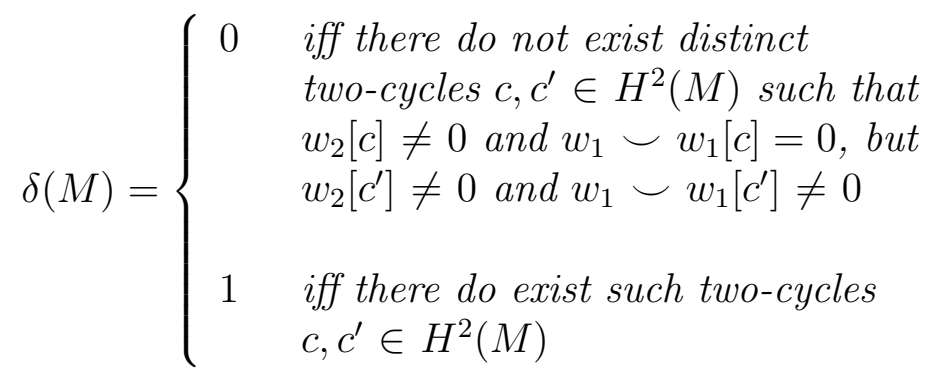

Now suppose we are given a manifold $M$ with boundary $\partial M$. Let $\tilde{M}$ and $\partial \tilde{M}$ denote the respective double covers. Then by Lemma 3, we have

$$
\begin{array}{r}
u(\partial M)+\chi(M)=w_{2}(M)+w_{1} \smile w_{1}(M) \bmod 2, \\
u(\partial \tilde{M})+\chi(\tilde{M})=w_{2}(\tilde{M}) \bmod 2 .
\end{array}
$$

Thus, we see that $\chi(M)+U(\partial M)=\chi(\tilde{M}) \bmod 2$ if and only if

$$
w_{2}(M)+w_{1}(M) \smile w_{1}(M)=w_{2}(\tilde{M}) \bmod 2 .
$$

We therefore obtain

Lemma 4. Let $M$ be a smooth non-orientable four-dimensional manifold with boundary. Let $\tilde{M}$ denote the oriented double cover of $M$, as above. Then

$$
U(\partial M)+\chi(M)=\chi(\tilde{M}) \bmod 2
$$


if and only if $\delta(M)=0$.

Proof. $(\Rightarrow)$ Suppose $U(\partial M)+\chi(M)=\chi(\tilde{M}) \bmod 2$, then $w_{2}(M)+$ $w_{1}(M) \smile w_{1}(M)=w_{2}(\tilde{M}) \bmod 2$. There are three cases to consider:

(i) $w_{2}(M)=w_{2}(\tilde{M})=0$

(ii) $w_{2}(M)=1$ and $w_{2}(\tilde{M})=0$

(iii) $w_{2}(M)=1=w_{2}(\tilde{M})$

If (i) holds, then $M$ and $\tilde{M}$ are both spin, and so we trivially have $\delta(M)=$ 0 .

If (ii) holds, then $\tilde{M}$ is spin, but $M$ is not. It follows that there exists some two-cycle $c \in H^{2}(M)$ such that $w_{2}[c] \neq 0$, and that this two-cycle lifts (under $\pi: \quad \tilde{M} \stackrel{2-1}{\longrightarrow} M)$ to a two-cycle $\tilde{c} \in H^{2}(\tilde{M})$ such that $w_{2}[\tilde{c}]=0$. Since $w_{2}[c]+w_{1} \smile w_{1}[c]=w_{2}[\tilde{c}]$ for any such two-cycle we must have $w_{1} \smile w_{1}[c]=1$ for all two-cycles $c \in H^{2}(M)$ such that $w_{2}[c]=1$. Thus we must have $\delta(M)=0$.

If (iii) holds, then neither $M$ nor $\tilde{M}$ is spin. Since $U(\partial M)+\chi(M)=$ $\chi(\tilde{M}) \bmod 2$, it follows that for all two-cycles $c \in H^{2}(M)$ such that $w_{2}[c] \neq 0$, we must have $w_{1} \smile w_{1}[c]=0$, and so $\delta(M)=0$.

$(\Leftarrow)$ Conversely, suppose that $\delta(M)=0$. Then case (i) gives $\chi(M)+$ $U(\partial M)=\chi(\tilde{M}) \bmod 2$ trivially.

Likewise, if (ii) holds, then on any of the two-cycles, $c$, for which $w_{2}[c]=1$, we must have $w_{1} \smile w_{1}[c]=1$, thus $\chi(M)+U(\partial M)=\chi(\tilde{M}) \bmod 2$.

Finally, case (iii) again implies that neither $M$ nor $\tilde{M}$ is spin. However, $\delta(M)=0$ again implies $w_{2}(\tilde{M})=w_{2}(M)+w_{1}(M) \smile w_{1}(M) \bmod 2$ and thus $\chi(M)+U(\partial M)=\chi(\tilde{M}) \bmod 2$.

Thus, $U(\partial M)+\delta(M) \in \mathbb{Z}_{2}$ is an invariant which tells us whether the Euler number of a manifold $M$ has the same parity as the Euler number of the double cover, $\tilde{M}$, of $M$. For convenience, we will henceforth write

$$
I(M, \partial M)=U(\partial M)+\delta(M)
$$

Suppose we are given some four-dimensional manifold $M$ with boundary $\partial M$ and its double cover $\tilde{M}$. Next form the 'double' [1] of each manifold, ie. we double $M$ to get $2 M$, and $\tilde{M}$ to get $2 \tilde{M} .2 \tilde{M}$ is then the oriented cover 
of $2 M$. As in [1], we have that

$$
\sigma(2 \tilde{M})=0,
$$

and so the index of any plane field $\tilde{P}$ on $2 \tilde{M}$ becomes

$$
\operatorname{ind}(\tilde{P}, 2 \tilde{M})=\frac{1}{4}(8 n-2 \chi(2 \tilde{M}), 8 m+2 \chi(2 \tilde{M}))
$$

where $m, n \in \mathbb{Z}$. We also know that

$$
\chi(2 \tilde{M})=2 \chi(\tilde{M}),
$$

and so we obtain

$$
\operatorname{ind}(\tilde{P}, 2 \tilde{M})=(2 n-\chi(\tilde{M}), 2 m+\chi(\tilde{M})) .
$$

We can assume that there are an equal number of singularities in each 'half' of the double $2 M$. As in [1], we then push all of the singularities over $\partial M$ into one of the halves. Then by construction, one of the halves of $2 M$ is free of singularities, and taking this half we have constructed a non-singular plane field on $M$. The degree of the map from $\partial M$ to $\tilde{G}_{2,4}$ (defined by the plane field) must be, combining equation (14) with Lemma 4,

$$
(2 n-\chi(M)+I(M, \partial M), 2 m+\chi(M)+I(M, \partial M)) \bmod 2
$$

As in [1], we shall call this degree the Klein kink of the metric $g_{K}$ (determining the plane field) with respect to $\partial M$ and we denote it

$$
\operatorname{kink}\left(\partial M ; g_{K}\right) \text {. }
$$

Combining the above, we obtain

Theorem 2. Let $M$ be any smooth four-dimensional manifold with boundary $\partial M \cong \Sigma_{1} \cup \Sigma_{2} \cup \cdots \cup \Sigma_{n} \neq \emptyset$, where $\left\{\Sigma_{i} \mid i=1, \ldots, n\right\}$ is some collection of closed three-manifolds. Then there always exist globally defined non-singular metrics $g_{K}$ of Kleinian signature on $M$. Furthermore every such metric must satisfy $\operatorname{kink}\left(\partial M ; g_{K}\right)=\left(k_{1}, k_{2}\right)$ where

$$
k_{i}=\chi(M)+I(M, \partial M) \bmod 2 \quad i=1,2 .
$$

Thus for an arbitrary non-singular Klein metric $g_{K}$ on $M$, the parity of the kink number of $g_{K}$ on $\partial M$ is completely determined. 


\section{Obstructions to pin structures}

As detailed in the Introduction, there are eight double covers of $O(2,2)$, which we denote

$$
h^{a, b, c}: \operatorname{Pin}^{a, b, c}(2,2) \longrightarrow O(2,2)
$$

where $a$ is the sign of the square of space inversion, $b$ is the sign of the square of time inversion, and $c$ is the sign of the square of the two combined. The obstructions to constructing a globally well-defined bundle, with fibre $\operatorname{Pin}^{a, b, c}(2,2)$, can be deduced using the constructions in [3]. Indeed, we obtain the following:

Theorem 3. Let $M$ be a Kleinian four-manifold (with tangent bundle $\tau(M)$ an $O(2,2)$ bundle). Then $M$ admits either $\operatorname{Pin}^{+,+,+}(2,2)$ or $\operatorname{Pin}^{+,+,-}(2,2)$ structure if and only if

$$
w_{2}(M)=0 .
$$

Theorem 4. Let $M$ be a Kleinian four-manifold (with tangent bundle $\tau(M)$ an $O(2,2)$ bundle). Then $M$ admits either $\operatorname{Pin}^{-,+,+}(2,2)$ or $\operatorname{Pin}^{-,+,-}(2,2)$ structure if and only if

$$
w_{2}(M)+w_{1}^{+} \smile w_{1}^{+}=0 .
$$

Theorem 5. Let $M$ be a Kleinian four-manifold (with tangent bundle $\tau(M)$ an $O(2,2)$ bundle). Then $M$ admits either $\operatorname{Pin}^{+,-,+}(2,2)$ or $\operatorname{Pin}^{+,-,-}(2,2)$ structure if and only if

$$
w_{2}(M)+w_{1}^{-} \smile w_{1}^{-}=0 .
$$

Theorem 6. Let $M$ be a Kleinian four-manifold (with tangent bundle $\tau(M)$ an $O(2,2)$ bundle). Then $M$ admits either $\operatorname{Pin}^{-,-,+}(2,2)$ or $\operatorname{Pin}^{-,-,-}(2,2)$ structure if and only if

$$
w_{2}(M)+w_{1}^{+} \smile w_{2}^{+}+w_{1}^{-} \smile w_{1}^{-}=0 .
$$


With these results, we can now investigate the obstructions to pin-Klein cobordism.

\section{Obstructions to pin-Klein cobordism}

In this section $\left\{\Sigma_{i} \mid i=1, \ldots n\right\}$ will always denote some collection of closed three-manifolds.

Definition: We will say that there exists a $\operatorname{Pin}^{a, b, c}(2,2)$ cobordism for $\left\{\Sigma_{i} \mid i=1, \ldots n\right\}$ if and only if there exists a Kleinian four-manifold $M$ (with a globally non-singular Kleinian metric $\left.g_{K}\right)$ admitting $\operatorname{Pin}^{a, b, c}(2,2)$ structure and satisfying

$$
\partial M \cong \Sigma_{1} \cup \Sigma_{2} \cup \ldots \cup \Sigma_{n}
$$

Corollary 1. There exists either a $\operatorname{Pin}^{+,+,+}(2,2)$ or a $\operatorname{Pin}^{+,+,-}(2,2)$ cobordism, $M$, for $\left\{\Sigma_{i} \mid i=1, \ldots n\right\}$ if and only if

$$
\left(u(\partial M)+k_{i}+I(M, \partial M)\right)=\left(w_{1}^{+} \smile w_{1}^{+}+w_{1}^{-} \smile w_{1}^{-}\right) \bmod 2
$$

where $k_{i}$ is either of the integers in $\operatorname{kink}\left(\partial M ; g_{K}\right)=\left(k_{1}, k_{2}\right)$.

Proof. $(\Rightarrow)$ Suppose such a pin-Klein cobordism, $M$, exists. Then by Theorem 2, we know that

$$
k_{i}=(\chi(M)+I(M, \partial M)) \bmod 2
$$

(since the Kleinian metric $g_{K}$ is non-singular). Furthermore, by Theorem 3, we must have

$$
w_{2}(M)=0,
$$

and by Lemma 3, we know that

$$
w_{2}(M)+w_{1}^{+} \smile w_{1}^{+}+w_{1}^{-} \smile w_{1}^{-}=(u(\partial M)+\chi(M)) \bmod 2 .
$$

Thus, combining equations (16), (17) and (18), we obtain

$$
\left(u(\partial M)+k_{i}+I(M, \partial M)\right)=\left(w_{1}^{+} \smile w_{1}^{+}+w_{1}^{-} \smile w_{1}^{-}\right) \bmod 2 .
$$


$(\Leftarrow)$ Conversely, suppose equation $(19)$ holds. Take any globally defined Klein metric $g_{K}$ on $M$, then we must have

$$
k_{i}=(\chi(M)+I(M, \partial M)) \bmod 2 .
$$

Hence $w_{2}=0$, and so $M$ is pin-Klein with the pin bundle fibre being $\operatorname{Pin}^{+,+, \pm}(2,2)$.

Using the above proof as a model, we also obtain:

Corollary 2. There exists either a $\operatorname{Pin}^{-,+,+}(2,2)$ or a $\operatorname{Pin}^{-,+,-}(2,2)$ cobordism, $M$, for $\left\{\Sigma_{i} \mid i=1, \ldots n\right\}$ if and only if

$$
\left(u(\partial M)+k_{i}+I(M, \partial M)\right)=w_{1}^{-} \smile w_{1}^{-} \bmod 2
$$

where $k_{i}$ is either of the integers in $\operatorname{kink}\left(\partial M ; g_{K}\right)=\left(k_{1}, k_{2}\right)$.

Corollary 3. There exists either a $\operatorname{Pin}^{+,-,+}(2,2)$ or a $\operatorname{Pin}^{+,-,-}(2,2)$ cobordism, $M$, for $\left\{\Sigma_{i} \mid i=1, \ldots n\right\}$ if and only if

$$
\left(u(\partial M)+k_{i}+I(M, \partial M)\right)=w_{1}^{+} \smile w_{1}^{+} \bmod 2
$$

where $k_{i}$ is either of the integers in $\operatorname{kink}\left(\partial M ; g_{K}\right)=\left(k_{1}, k_{2}\right)$.

Corollary 4. There exists either a $\operatorname{Pin}^{-,-,+}(2,2)$ or a $\operatorname{Pin}^{-,-,-}(2,2)$ cobordism, $M$, for $\left\{\Sigma_{i} \mid i=1, \ldots n\right\}$ if and only if

$$
\left(u(\partial M)+k_{i}+I(M, \partial M)\right)=0 \bmod 2
$$

where $k_{i}$ is either of the integers in $\operatorname{kink}\left(\partial M ; g_{K}\right)=\left(k_{1}, k_{2}\right)$.

Thus we see that the obstructions to $\operatorname{Pin}^{a, b, c}(2,2)$ cobordism depend only on boundary data (ie. $u(\partial M)$ and $\left.\operatorname{kink}\left(\partial M ; g_{K}\right)=\left(k_{1}, k_{2}\right)\right)$, the values of $a, b \in\{ \pm\}$, the choice of orientation (ie. $w_{1}^{+} \smile w_{1}^{+}$and $w_{1}^{-} \smile w_{1}^{-}$) and the invariant $I(M, \partial M)$.

Finally, we note that in all the above Corollaries, the expression $u(\partial M)+$ $k_{i}+I(M, \partial M)$ may be replaced by the expression $u(\partial \tilde{M})+k_{i}+\delta(M)$, since $I(M, \partial M)=U(\partial M)+\delta(M)=(u(\partial M)+u(\partial \tilde{M})) \bmod 2+\delta(M)$.

\section{Examples and Applications}


The constuctions introduced in this paper have many applications to theoretical physics. We now give some examples and applications.

Example 1. Let $K$ denote the Klein bottle and $T \cong S^{1} \times S^{1}$ the Torus. Then form a Kleinian metric on $M \cong K \times T$ by taking the product metric formed by using the natural negative definite metric on $K$ and the natural positive definite metric on $T$. Although $M$ is non-time orientable (since traversing the orientation-reversing loop in $K$ inverts the timelike sub-bundle), $M$ is still spin since $w_{2}(K)=0$, and so all $\operatorname{Pin}^{a, b, c}(2,2)$ structures are allowed on $M$.

Now suppose that we take $M$ to be the product space $M \cong \mathbb{R P}^{2} \times T$ where, as above, we endow $M$ with the natural product metric such that $\mathbb{R P}^{2}$ is timelike. Then we clearly have $w_{1}^{-} \smile w_{1}^{-}\left(\mathbb{R P}^{2}\right)=1$ and $w_{2}(M)=1 \bmod 2$. Thus not all pin structures will be allowed. Indeed, one easily calculates that $\operatorname{Pin}^{+,+, \pm}(2,2)$ and $\operatorname{Pin}^{-,+, \pm}(2,2)$ structures will not be allowed, whilst $\operatorname{Pin}^{+,-, \pm}(2,2)$ and $\operatorname{Pin}^{-,-, \pm}(2,2)$ are allowed.

Example 2. An interesting application is to Kaluza-Klein type theories in which some of the internal dimensions are allowed to be timelike. We could take the ground state of such a theory to be a manifold of the form $M \times S^{1}$ where $M$ is a Lorentzian three-manifold, and the internal space $S^{1}$ is timelike. Then the total metric on $M \times S^{1}$ would have signature --++ , and the obstruction to this metric being non-singular would again be the condition that there exists a plane field. We could even allow the Kleinian metric to 'spin around', so that the internal space fluctuates from being timelike to being spacelike ( so that the signature of the spacetime $M$ would change from -++ to +--$)$. That is, in terms of the effective three-dimensional theory, this would correspond to 'signature change'.

In general, in order to produce a non-singular theory, we may wish to consider manifolds $M$ with Kleinian, or even more exotic, signatures. For example, if $M \cong S^{2} \times S^{2}$ then $M$ does not admit a non-singular Lorentz metric, but does admit a non-singular Klein metric. Such choices will generically change the types of Pin structures which are admitted in the Kaluza-Klein (or other) type theory.

Example 3. There has been considerable interest recently in the study of signature changing spactimes [5-8]. In an extension to the example given in [1] we note that we can have the nucleation of a single Kleinian region across 
a single zero-kink surface homeomorphic to $S^{3}$. As an example, let $M$ be the Kleinian manifold formed by removing a four-ball from $\mathbb{R P}^{2} \times S^{2}$ such that the $S^{2}$ factor is timelike. We have $\partial M \cong S^{3}$, and so $I(M, \partial M)=0$. In order to produce signature change we require that the kink on $\partial M$ is zero. Then since $u\left(S^{3}\right)=1$, we see that such a signature change scenario is possible since $M$ admits a $\operatorname{Pin}^{-,+, \pm}(2,2)$ structure.

Example 4. Finally, we note that our results would be potentially useful in generalising the Penrose flag-plane construction [16] to non-orientable manifolds. 


\section{Acknowledgements}

The authors thank Gary Gibbons for many helpful discussions. Thanks also to Sylvia Fouet for translating most of reference [12] into English, and to Jo Chamblin for helping prepare this paper. A.C. is supported by N.S.F. Graduate Fellowship No. RCD-9255644.

\section{References}

[1] L. J. Alty and A. Chamblin, Spin Structures on Kleinian Manifolds, Class. Quantum Grav. 11, 2411-2415 (1994).

[2] L. Dabrowski, Group Actions on Spinors, Monographs and Textbooks in Physical Science, Bibliopolis (1988).

[3] A. Chamblin, On the Obstructions to non-Cliffordian Pin Structures, Comm. Math. Phys. 164, 65-87 (1994).

[4] F. Wilczek, Fractional Statistics and Anyon Superconductivity, World Scientific (1990).

[5] G. Ellis, A. Sumeruk, D. Coule and C. Hellaby, Change of signature in classical relativity, Class. Quantum. Grav. 9 1535-1554 (1992).

[6] S. A. Hayward, Signature change in general relativity, Class. Quantum. Grav. 9 1851-1862 (1992).

[7] T. Dray, C. A. Manogue and R. W. Tucker, Scalar Field Equation in the presence of Signature Change, Phys. Rev. D 48, 2587-2590 (1993).

[8] L. J. Alty, Kleinian Signature Change, Class. Quantum Grav. 11, 25232536 (1994).

[9] J. Barret, G. W. Gibbons, M. J. Perry, C. N. Pope, and P. Ruback, Kleinian Geometry and the $N=2$ Superstring, Int. J. Mod. Phys. A 9 1457-1493 (1994).

[10] N. Steenrod, Topology of Fibre Bundles, Princeton Math. Series, Princeton Univ. Press (1951).

[11] A. Chamblin, Some Applications of Differential Topology in General 
Relativity, J. Geom. Phys. 13, 357-377 (1994).

[12] F. Hirzebruch and H. Hopf, Felder von flächenelementen in 4-dimensionalen mannigfaltigkeiten, Math. Ann. 136, 156-172 (1958).

[13] E. Thomas, Vector Fields on Manifolds, Bull. Amer. Math. Soc. 75, 643-668 (1969).

[14] J. W. Milnor and J. D. Stasheff, Characteristic Classes, Princeton Univ. Press (1974).

[15] M. Kervaire and J. W. Milnor, Groups of Homotopy Spheres: I, Ann. of Math. 77, (1963).

[16] R. Penrose and W. Rindler, Spinors and Space-time - Volume 1, Cambridge University Press (1986). 
This figure "fig1-1.png" is available in "png" format from: http://arxiv.org/ps/gr-qc/9505004v1 
This figure "fig1-2.png" is available in "png" format from: http://arxiv.org/ps/gr-qc/9505004v1 